while the completion of the interferometer is foreseen for 1986 only. This is determined by the possible inflow of funds and not by technical considerations. However, since the interferometer consists of three antennas, it is foreseen to proceed in stages, such that the first antenna at Plateau de Bure can be made operational by 1983 .

\section{Present Status}

The negotiations with Spain on the Sierra Nevada site are in the final stage. At first it was hoped to place the antenna very close to the Pico de Veleta (at $3300 \mathrm{~m}$ ), but this met environmental objections. A satisfactory site near-by (at $2900 \mathrm{~m}$ ) has been offered instead. In exchange for offering a site and the local headquarters in Granada, Spanish astronomers will get about $10 \%$ observation time at both observatories, and will become an integrated part of the radio astronomy community utilizing the instruments.
The MPIfR are almost ready to place the order for the construction of the large dish and the design of the interferometer antennas is in the initial stage. Here the first task is to determine the final diameter to fit the allocated funds.

The project office will be established in Grenoble in May this year, in a temporary building put at IRAM's disposal by the University of Grenoble. By the end of 1979 , it is hoped that IRAM will have 20-25 people in Grenoble, with several technical groups active, in particular, the receiver group.

\section{Concluding Remarks}

Another international research institute is in the process of being born. It has for the time being only two funding partners, which constitute both advantages and disadvantages. It is to be hoped that more partners will join later to make IRAM a truly European Institute.

\title{
TeV Fixed Target Proton Synchrotron Projects
}

A shortened version of the paper prepared by K. P. Myznikov of Moscow in the name of a team of over $\mathbf{5 0}$ physicists from institutes in the USSR. At the seminar, the paper was introduced by $\mathrm{V}$. Sidorov.

The Institute for High Energy Physics' Accelerating and Storage Complex (UNK), being developed for still deeper studies in high energy physics is a $3 \mathrm{TeV}$ superconducting proton synchrotron with the prospect of obtaining colliding beams in the future. The design study has already been

Lay-out of UNK in relation to the present Serpukhov machine.

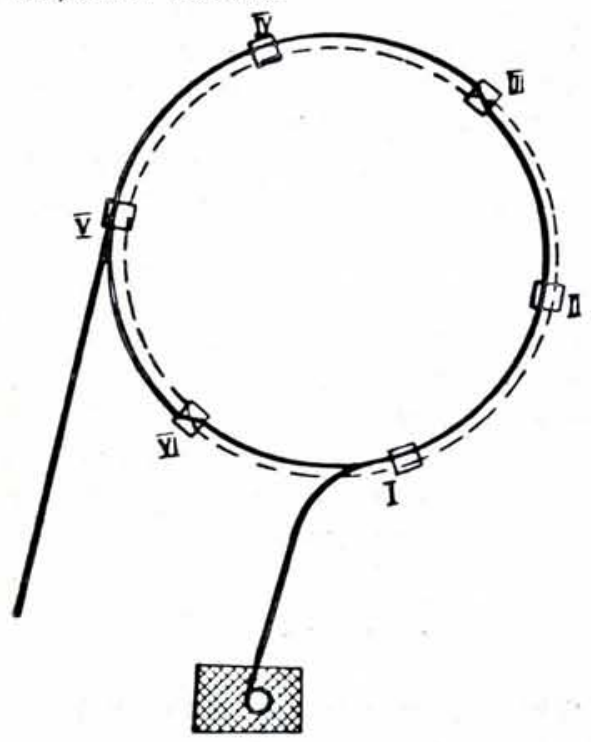

completed, and the basic parameters are listed in Table I.

UNK is a two-stage accelerator, wherein the first stage is a proton synchrotron with conventional magnets, designed to stack and pre-accelerate protons up to $400 \mathrm{GeV}$, and the second an accelerator utilizing superconducting magnets for achieving the highest energies. Both accelerators are located in a common tunnel. The existing $70 \mathrm{GeV}$ accelerator at Serpukhov (U-70), the intensity of which is planned to be increased up to $5 \times 10^{13} \mathrm{ppp}$, is to be used as an injector into UNK.

The two-stage system simplifies the superconducting accelerator operating mode substantially. Beam preacceleration in the first stage makes it possible to reduce the effects of residual fields, and the decrease in beam dimensions reduces the severity of the field specifications imposed on the superconducting magnets. This is also helped by having single-turn beam injection into the central orbit of the second stage. Beam stacking takes place in the first stage, so freeing the superconducting accelerator from inevitable particle losses.
Other advantages are to be found in the two-stage system. It allows the maximum use to be made of the injector intensity and the availability of two rings leaves open the option of a $0.4 \mathrm{TeV}$ and $3 \mathrm{TeV}$ proton-proton colliding beam facility yielding 2.2 $\mathrm{TeV}$ energy in the centre of mass. The ring of the first stage can also be used to stack pure beams of electrons, antiprotons, etc.

Fig. 1 shows the layout of UNK, with the main ring centre, taking into account geological restraints, located $6.5 \mathrm{~km}$ from the U-70 accelerator centre. Dimensions of the tunnel are chosen with in mind the possibility of installing there a superconducting storage ring. This will enable a $3 \mathrm{TeV}$ proton beam to be stacked and made to collide with a beam of the same energy accelerated in the pulse superconducting ring. As a result, the ppcollision energy can be increased up to $6 \mathrm{TeV}$ in the c.m.s.

The UNK project is designed with its future development in view. Six straight sections, each $485 \mathrm{~m}$ long, are introduced into the structure of both stages (Fig. 1). A beam from $\mathrm{U}-70$ is injected into section I, where the beam transfer system into the second stage is also located. The accelerated particles are extracted from section V. The accelerating systems are placed in section II, and the beam abort systems in section IV. Sections III and IV are reserved for colliding beams. The layout of the accelerator and storage rings in the tunnel and the construction of the straight sections are chosen so that the intersections of the maximum energy beams would be obtained more easily. Fig. 2 presents a schematic view of the UNK tunnel cross-section and the position of the magnets there.

Great importance has been attached to developing for the superconducting magnet units protection measures against radiation. Stacking at $70 \mathrm{GeV}$ and beam pre-acceleration take place in the first stage

Cross-section of the tunnel showing: 1 , first stage; 2 , second; 3 , storage ring.

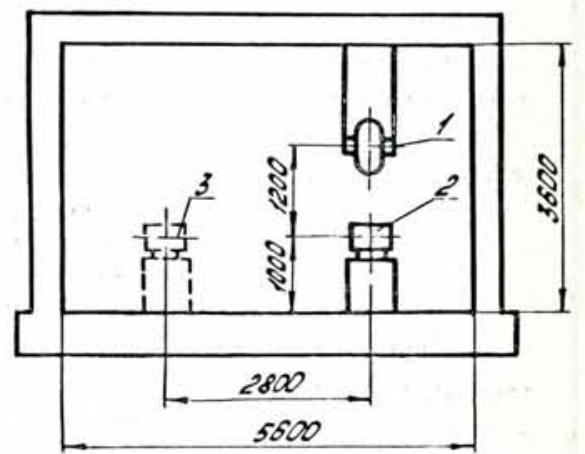


Table I - Main Parameters of UNK.

\begin{tabular}{lccc}
\hline Name & $\begin{array}{c}\text { Unit of } \\
\text { Measurement }\end{array}$ & $\begin{array}{c}\text { First } \\
\text { Stage }\end{array}$ & $\begin{array}{c}\text { Second } \\
\text { Stage }\end{array}$ \\
\hline Total length & $\mathrm{m}$ & 19288 & 19288 \\
Injection energy & $\mathrm{GeV}$ & 70 & 400 \\
Maximum energy & $\mathrm{GeV}$ & 400 & 3000 \\
Injection field & $\mathrm{T}$ & 0.117 & 0.670 \\
Maximum magnetic field & $\mathrm{T}$ & 0.670 & 5.0 \\
Cycle duration & $\mathrm{s}$ & 78 & 78 \\
Pulse intensity & $\mathrm{p} /$ cycle & $6 \times 10^{14}$ & $6 \times 10^{14}$ \\
\hline
\end{tabular}

decreasing substantially the space charge effects in the second stage. In addition, various methods of beam scraping will be applied in the first stage facilitating an efficient (close to $100 \%$ ) beam transfer into the second stage. To localize possible beam losses during acceleration in both stages, beam scraping stations are arranged in the long straight sections. Their length makes it possible to put in the necessary number of collimators after these stations and to suppress particle fluences arising there, down to the level suitable for the normal operation of the superconducting magnets. Should inadmissibly large beam losses occur, the accelerated beam will be extracted from the accelerator during one turn, with the help of beam abort systems, into the outer stoppers.

The operational cycle of UNK is the following. Successive beams of $5 \times 10^{13}$ protons are accelerated in the $U-70$ by an RF field that has a frequency of $200 \mathrm{MHz}$, equal to the UNK accelerating voltage frequency. Stacking of $6 \times 10^{14} \mathrm{ppp}$ during $71.5 \mathrm{~s}$ is achieved by successive injection of 12 pulses from the U-70. The UNK circumference is exactly 13 times as long as that of the U-70, so that part of the first stage circumference is not filled with beam. This makes it possible to arrange "time slots" between the beam pulses, necessary to facilitate the operation of injection and extraction devices and thus to reduce particle losses. On completion of stacking, the beam is accelerated in the first stage up to $400 \mathrm{GeV}$ and single-turn transferred into the second. The second stage comprises $20 \mathrm{~s}$ acceleration, $38 \mathrm{~s}$ beam extraction and $20 \mathrm{~s}$ field decay time.

Provision is made for correction stations to be installed in the long matched straight sections. To adjust betatron frequencies, chromaticity and equilibrium orbit, it is intended to position near the main quadrupoles universal superconducting correctors fitted with dipole, quadrupole and sextupole coils. Orbit correction is envisaged in both oscillation planes. 180 correctors working in either plane and with $0.5 \mathrm{~mm}$ r.m.s. orbit measurement resolution, the design correction accuracy is $\pm 2 \mathrm{~mm}$. As a result maximum beam dimensions at injection into the first stage are $50 \mathrm{~mm}$ in the vertical direction and $70 \mathrm{~mm}$ in the horizontal plane and during injection into the second stage $-42 \mathrm{~mm}$ and $50 \mathrm{~mm}$, respectively. The dimensions of the vacuum
Among many other models, theorists Weinberg and Salam proposed, independently at the end of the sixties a model for the unification of weak and electromagnetic interactions. This model requires the existence of neutral weak currents. They were discovered in a neutrino beam at CERN in 1973 and 1974.

At the same time, S.S. Ting using a proton beam at the AGS of Brookhaven and $B$. Richter running the $\mathrm{e}^{+} \mathrm{e}^{-}$ collider (SPEAR) at Stanford, discovered the $\mathrm{J} / \psi$ particle. $\mathrm{J} / \psi$ is considered as composed of a pair of one charmed quark and an antiquark. It is now generally admitted that hadrons exhibit at high mementum transfer, above a few $\mathrm{GeV}^{2}$, a substructure and seem to be made of quarks and antiquarks.

Since 1974 , subnuclear physics has known a very exciting period with new discoveries made with the large synchrotrons at Fermilab and CERN and with the $\mathrm{e}^{+} \mathrm{e}^{-}$colliders at Stanford and first DORIS then from the middle of 1978 with PETRA at DESY.

However, to establish the validity of the Weinberg and Salam model definitively, one has to discover the two charged vector bosons $\mathrm{W} \pm$ with chamber in the first stage are of two types, $47 \times 87 \mathrm{~mm}^{2}$, and $65 \times 65 \mathrm{~mm}^{2}$; the chamber of the second stage has an elliptical cross section of $60 \times$ $70 \mathrm{~mm}^{2}$.

Vacuum requirements in the chambers of the two UNK stages are determined by proton losses due to the residual gas. Mean pressure in the first stage should not exceed $3 \times 10^{-7}$ torr nitrogen equivalent and $2 \times 10^{-8}$ torr in the second. Preliminary calculations show that with the 20$40 \mathrm{~K}$ temperature of the chamber walls of the superconducting ring, a $2 \times 10^{-11}$ torr vacuum can be obtained for hydrogen and $\sim 10^{-13}$ torr for other gases.

The UNK accelerating system has to provide $12 \mathrm{MV}$ total amplitude at $200 \mathrm{MHz}$ for the first stage and $17 \mathrm{MV}$ for the superconducting ring, with maximum powers respectively of 6.6 $\mathrm{MW}$ and $18 \mathrm{MW}$.

\section{Large Electron-Positron Colliding Beam Project}

Based on a summary of his paper prepared by M. Vivargent, chairman of ECFA, that traces the rapid development of high energy physics since large machines became available for experiments. LEP is western Europe's choice for the next generation.

a mass of about $78 \mathrm{GeV}$ and the $Z^{\circ}$ meson with a mass of about $90 \mathrm{GeV}$, coupled respectively to the weak charges and the neutral currents. Their model also predicts the existence of a very special boson called the "Higgs" boson.

Since 1976, ECFA - the European Committee for Future Accelerators a body representing the physics community of the CERN Member States has carefully examined what accelerators should be built to maintain European subnuclear physics at the highest international level at the end of the eighties.

To solve the problems arising from the attempt to unify weak and electromagnetic interactions and from the development of a new theory of strong interactions called Q.CD, phycists of western Europe have unanimously proposed the construction of a large electron-positron collider called LEP.

CERN was asked by ECFA in May 1977 , to study a $\mathrm{e}^{+} \mathrm{e}^{-}$collider with an energy up to $100 \mathrm{GeV}$ per beam possibly located near the existing CERN site.

Early studies revealed great technical difficulties and heavy financial implications for the construction of a 